\title{
CT angiography helps to differentiate acute from chronic carotid occlusion: the "carotid ring sign"
}

\author{
Patrik Michel • George Ntaios • \\ Montserrat G. Delgado • Daniel C. Bezerra • \\ Reto Meuli $\cdot$ Stefano Binaghi
}

Received: 20 December 2010 /Accepted: 28 March 2011 /Published online: 12 April 2011

(C) Springer-Verlag 2011

\begin{abstract}
Introduction Currently, there is no reliable method to differentiate acute from chronic carotid occlusion. We propose a novel CTA-based method to differentiate acute from chronic carotid occlusions that could potentially aid clinical management of patients.

Methods We examined 72 patients with 89 spontaneously occluded extracranial internal carotids with CT angiography (CTA). All occlusions were confirmed by another imaging modality and classified as acute (imaging $<1$ week of presumed occlusion) orchronic (imaging $>4$ weeks), based on circumstantial clinical and radiological evidence. A neuroradiologist and a neurologist blinded to clinical information determined the site of occlusion on axial sections of CTA. They also looked for (a) hypodensity in the carotid artery (thrombus), (b) contrast within the carotid wall (vasa vasorum), (c) the site of the occluded carotid,
\end{abstract}

P. Michel $\cdot$ G. Ntaios $(\varangle)$

Neurology Service, Centre Hospitalier Universitaire

Vaudois and University of Lausanne,

Rue De Bugnon 46,

CH 1011 Lausanne, Switzerland

e-mail: ntaiosgeorge@yahoo.gr

M. G. Delgado

Hospital Universitario Central de Asturias,

Oviedo, Spain

D. C. Bezerra

Department of Epidemiology,

Johns Hopkins Bloomberg School of Public Health,

Baltimore, MD, USA

R. Meuli $\cdot$ S. Binaghi

Department of Radiology, Centre Hospitalier Universitaire

Vaudois and University of Lausanne,

Lausanne, Switzerland and (d) the "carotid ring sign" (defined as presence of a and/or b).

Results Of 89 occluded carotids, 24 were excluded because of insufficient circumstantial evidence to determine timing of occlusion, 4 because of insufficient image quality, and 3 because of subacute timing of occlusion. Among the remaining 45 acute and 13 chronic occlusions, inter-rater agreement (kappa) for the site of proximal occlusion was $0.88,0.45$ for distal occlusion, 0.78 for luminal hypodensity, 0.82 for wall contrast, and 0.90 for carotid ring sign. The carotid ring sign had $88.9 \%$ sensitivity, $69.2 \%$ specificity, and $84.5 \%$ accuracy to diagnose acute occlusion.

Conclusion The carotid ring sign helps to differentiate acute from chronic carotid occlusion. If further confirmed, this information may be helpful in studying ischemic symptoms and selecting treatment strategies in patients with carotid occlusions.

Keywords Carotid ring sign - Internal carotid occlusion . CT angiography

Stroke due to acute internal carotid artery (ICA) occlusion usually has severe long-term consequences [1], and responds poorly to intravenous thrombolysis [2,3]. Acute thromboendarterectomy yielded poor results in older studies [4], but recent case series of acute endovascular treatments suggest benefits in selected patients [5-10]. Rapid diagnosis of carotid artery occlusion and correct classification as acute versus chronic has therefore become of clinical importance but remains difficult in the absence of a reliable diagnostic method.

We observed that carotid occlusions in patients with acute ipsilateral hemispheric stroke frequently showed on axial images on CT angiography (CTA), a ring of contrast enhancement in the carotid wall surrounding a hypodense 
thrombus in the lumen (Fig. 1). We hypothesized that the findings in this image, which we termed carotid ring sign, could accurately differentiate between acute and chronic carotid occlusions. The present study aims to test this hypothesis.

\section{Methods}

\section{Patients}

The majority (78\%) of acute ischemic stroke patients admitted to our institution are investigated with CTA, and reveals a significant vascular pathology in $47.2 \%$ [11]. For this analysis, we retrospectively screened all patients admitted to our hospital between May 2000 and July 2005 with an acute ischemic cerebrovascular event. We selected patients with an extracranial internal carotid occlusion diagnosed by CTA and confirmed by a second imaging technique (i.e., carotid Doppler ultrasound, magnetic resonance angiography (MRA) or classical angiography) within 7 days of the CTA. Patients were excluded from the study when (a) the quality of CTA images was poor, (b) there was insufficient evidence to allow classification of the carotid occlusion as "acute" or "chronic" according to the criteria listed below, or (c) the occlusion likely occurred in an intermediate phase, i.e., 7-30 days before CTA. These cut offs were arbitrarily chosen to increase the likelihood of finding reliable diagnostic criteria on CTA.

\section{CTA technique and image evaluation}

In our institution, the parameters for cerebral and cervical CTA acquired by multidetector-array technology in helicoidal

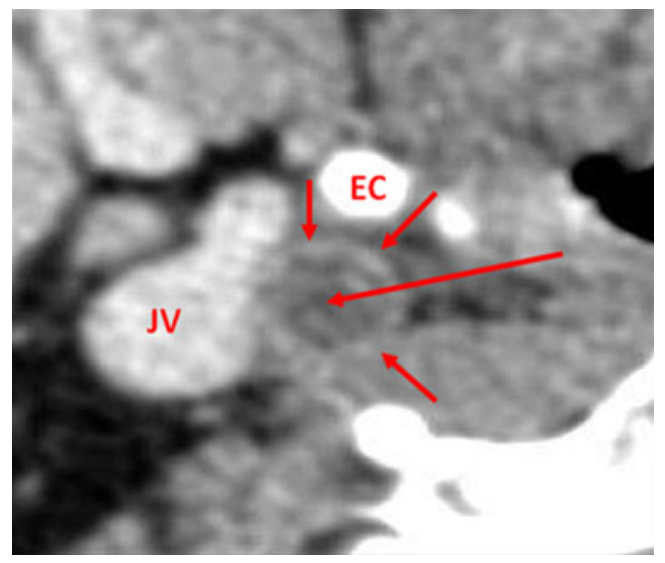

Fig. 1 Axial image on cervical CTA of the right internal carotid of a 67-year-old patient with an acute atherosclerotic stroke at the middle cerebral artery territory. CTA was performed at the day of symptom onset. An occluded internal carotid with a hypodense lumen (long arrow) and a ring of contrast enhancement at the vascular wall (short arrows) is shown. $E C$ external carotid, $J V$ jugular vein mode during the observation period were $140 \mathrm{kV}, 260 \mathrm{mAs}$, slice thickness $1.25 \mathrm{~mm}$, pitch 0.9:1. Acquisition delay depends on the bolus test of $20 \mathrm{ml}$ (range 15-20 s). Acquisition is then performed after intravenous administration of $50 \mathrm{ml}$ of iohexol $(300 \mathrm{mg} / \mathrm{ml}$ of iodine; Accupaque 300 , Amersham, Oslo, Norway) at a rate of $5 \mathrm{ml}$ per second with a power injector (Stellant ${ }^{\circledR}$ D CT Injection System; Medrad, Warrendale, PA, USA). Data acquisition is performed from the origin of the aortic arch branch vessels to the frontal sinuses. Maximum-intensity projections are then reconstructed in axial, sagittal, and coronal planes.

A neuroradiologist $(\mathrm{SB})$ and a neurologist $(\mathrm{PM})$ that were blinded to patients and to clinical information independently determined the site where the occlusion started with relationship to the extracranial carotid bifurcation, the site where it ended in intracranial portion of the internal carotid, and the length of the occlusion on axial sections of the CTA. In addition, they looked for the presence of hypodensity in the lumen of the occluded carotid (corresponding to the occlusive thrombus) and contrast within the carotid wall. The carotid ring sign was defined as the presence of ring-like contrast enhancement in the carotid wall and/or intraluminal hypodensity (Fig. 1). In case of discordance between the two readers, a consensus was reached.

\section{Classification of ICA occlusion}

In the absence of a gold standard for differentiating acute from chronic carotid occlusion, we chose to use circumstantial evidence such as classification, the type and localization of the acute cerebrovascular events, prior carotid imaging, and autopsy findings. Extracranial ICA occlusions were classified with regards to the time that occurred as acute when the estimated time of occlusion was within 7 days prior to CTA or chronic when more than 30 days before. They were also classified with regards to the level of certainty for the timing of occlusion as certain or probable, according to circumstantial evidence.

An acute extracranial ICA occlusion was classified as certain when (a) confirmed as such by autopsy, (b) anarterial imaging study $<7$ days earlier showing patency, (c) caused by a dissection with associated neurological symptoms (e.g., Horner syndrome, transient ischemic attack (TIA), stroke), or (d) a follow-up arterial imaging study demonstrated recanalization. On the other hand, it was classified as probable in the case of symptomatic ischemic stroke confirmed by a new parenchymal lesion in the territory of the occluded carotid (except when there was a $\geq 70 \%$ stenosis of another major artery serving as potential collateral and embolic source to the occluded carotid territory, i.e., contralateral internal carotid, ipsilateral external carotid, basilar artery). 
A chronic extracranial ICA occlusion was classified as certain when (a) confirmed as such by autopsy, or (b) an occlusion was previously documented by an arterial imaging study, and there was no dissection or cardioembolic source. On the other hand, it was classified as probable when (a) an occlusion that was due to dissection or cardioembolism was previously documented by an arterial imaging study, (b) it was an incidental finding in an asymptomatic patient (without any recent ( $\geq 1$ month) cerebrovascular event) and without an acute/subacute parenchymal lesion on CT or MRI in the territory of the occluded carotid, or (c) it was an incidental finding in a symptomatic patient (with recent ischemic cerebrovascular event outside the territory of the occluded carotid) without a $>70 \%$ stenosis in another major artery serving as potential collateral to the occluded carotid artery (contralateral internal carotid, ipsilateral external carotid, basilar artery).

\section{Statistical analysis}

Cohen's kappa ( $\mathrm{k}$ ) statistical analysis for inter-rater agreement was performed to evaluate the degree of agreement between the two readers. Sensitivity, specificity, accuracy, positive and negative predictive value (PPV and NPV, respectively), and positive and negative likelihood ratio ( $\mathrm{LR}+$ and $\mathrm{LR}^{-}$) of the luminal hypodensity, the wall contrast enhancement, their combination and the carotid ring sign were calculated from the true positive (TP), true negative (TN), false positive (FP), and
Fig. 2 Flow diagram of the analysis

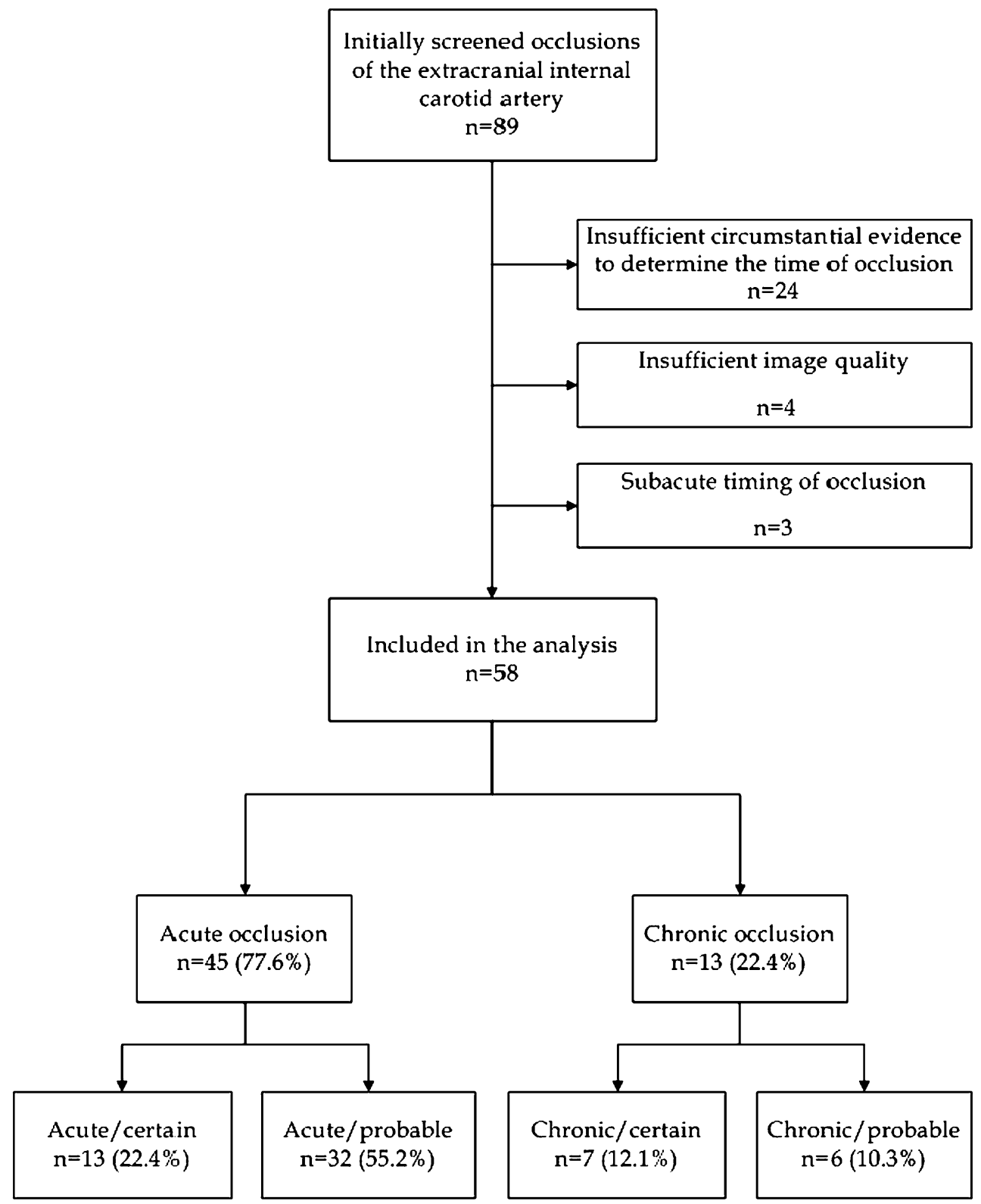


false negative $(\mathrm{FN})$ readings using $2 \times 2$ contingency tables and two-tailed chi-squared test.

\section{Results}

We identified 89 cervical CTA examinations with occlusion of the extracranial ICA between May 2000 and July 2005.
Of them, 31 were excluded (24 due to insufficient circumstantial evidence, 5 due to poor image quality, and 3 due to subacute timing of the occlusion) and the remaining 58 occlusions (in 50 different patients) were classified as acute or chronic, and further stratified according to the level of certainty for the estimation of the timing of the occlusion (Fig. 2). In four cases, the estimation of the two reviewers about the presence of the sign was different
Table 1 Characteristics of the 58 carotid occlusions included in the final analysis
The numbers represent count (percentage) for categorical variables and mean \pm standard deviation for age

\begin{tabular}{|c|c|c|}
\hline Characteristics & $\begin{array}{l}\text { Acute occlusion } \\
n=45\end{array}$ & $\begin{array}{l}\text { Chronic occlusion } \\
n=13\end{array}$ \\
\hline \multicolumn{3}{|l|}{ Side of occlusion } \\
\hline Right & $27(60.0 \%)$ & $3(23.1 \%)$ \\
\hline Left & $18(40.0 \%)$ & $8(61.6 \%)$ \\
\hline Bilateral & - & $1(7.7 \%)$ \\
\hline \multicolumn{3}{|l|}{ Most proximal site of occlusion } \\
\hline Common carotid artery & $2(4.5 \%)$ & - \\
\hline Bifurcation & $29(64.4 \%)$ & $11(84.6 \%)$ \\
\hline Above bifurcation $(>1 \mathrm{~cm})$ & $14(31.1 \%)$ & $2(15.4 \%)$ \\
\hline \multicolumn{3}{|l|}{ Most distal site of occlusion } \\
\hline Proximal siphon-cavernous segment & $20(44.4 \%)$ & $3(23.1 \%)$ \\
\hline Distal siphon-supraclinoid segment & $15(33.3 \%)$ & $8(61.5 \%)$ \\
\hline Below siphon & $9(19.9 \%)$ & $1(7.7 \%)$ \\
\hline Movement artifact at this site & $1(2.2 \%)$ & $1(7.7 \%)$ \\
\hline \multicolumn{3}{|l|}{ Mechanism of occlusion } \\
\hline Atherosclerosis & $19(42.2 \%)$ & $10(76.9 \%)$ \\
\hline Cardioembolism & $9(20.0 \%)$ & $1(7.7 \%)$ \\
\hline Dissection & $8(17.7 \%)$ & - \\
\hline Radiation & $1(2.2 \%)$ & - \\
\hline Sphenoidal osteomyelitis & $1(2.2 \%)$ & - \\
\hline Paraneoplastic hypercoagulability & $2(4.5 \%)$ & - \\
\hline Undetermined & $7(15.6 \%)$ & $2(14.4 \%)$ \\
\hline \multicolumn{3}{|l|}{ Reason for CTA } \\
\hline Acute stroke & $43(95.5 \%)$ & $6(46.1 \%)$ \\
\hline Acute transient ischemic attack & $2(4.5 \%)$ & $5(38.5 \%)$ \\
\hline Chronic stroke & - & $1(7.7 \%)$ \\
\hline Confusion - sepsis & - & $1(7.7 \%)$ \\
\hline Age of the patient (years) & $68.5 \pm 21.8$ & $68.6 \pm 9.1$ \\
\hline Gender of the patient (female) & $22(57.9 \%)$ & $\begin{array}{l}2(16.7 \%) \\
(16.7 \%)\end{array}$ \\
\hline \multicolumn{3}{|l|}{ Medical history of the patient } \\
\hline Previous ischemic cerebrovascular event & $13(34.2 \%)$ & $5(41.7 \%)$ \\
\hline Arterial hypertension & $29(76.3 \%)$ & $9(75 \%)$ \\
\hline Diabetes mellitus & $11(28.9 \%)$ & $2(16.7 \%)$ \\
\hline Active smoking & $11(28.9 \%)$ & $4(33.4 \%)$ \\
\hline Dyslipidemia & $23(60.5 \%)$ & $7(58.4 \%)$ \\
\hline Atrial fibrillation & $10(26.4 \%)$ & $4(33.4 \%)$ \\
\hline Coronary artery disease & $5(13.2 \%)$ & $3(23.1 \%)$ \\
\hline Biological or mechanical heart valves & $1(2.6 \%)$ & - \\
\hline Low ejection fraction $(<30 \%)$ & $5(13.2 \%)$ & - \\
\hline
\end{tabular}


and a consensus was reached. Among the 50 patients, 1 had a bilateral carotid occlusion (both occlusions included in the analysis), 5 patients had two consecutive CTAs for a single occlusion (both CTAs included in the analysis), and 1 patient had three consecutive CTAs for a single occlusion (all three CTAs included in the analysis). The baseline characteristics of the occlusions are summarized in Table 1. The initially CTA-diagnosed occlusions were confirmed by cervical Doppler ultrasound in all 58 cases, MRA in 21 $(36.2 \%)$, angiography in $2(3.4 \%)$, and autopsy in $1(1.7 \%)$. Eleven $(18.9 \%)$ patients were treated with intravenous thrombolysis (Table 1).

Cohen's k was 0.88 for the inter-rater agreement for the site of proximal occlusion, 0.45 for the site of distal occlusion, 0.70 for the luminal hypodensity, 0.82 for the contrast within the wall, and 0.86 for the carotid ring sign.

Table 2 summarizes the sensitivity, specificity, accuracy, PPV, and NPV of the presence of luminal hypodensity or wall contrast or their combination or the carotid ring sign to diagnose an acute extracranial ICA occlusion. The statistical difference noted for cross tabulation between the presence and absence of each sign was significant $(p<0.01$ for the presence of luminal hypodensity, wall contrast or their combination, and $p<0.05$ for the carotid ring sign). The carotid ring sign was the index with the highest accuracy (84.5\%), sensitivity (88.9\%), and NPV (64.3\%), whereas the presence of wall contrast enhancement showed the highest specificity (84.6\%) and PPV (94.9\%).

The employment of the carotid ring sign yielded four false-positive and five false-negative results. The four-false positive results were obtained in three patients classified as having chronic occlusions: (a) a patient with a stroke at the distribution of the middle cerebral artery (MCA) during carotid endarterectomy (CEA) for symptomatic right ICA stenosis with an incidental left ICA occlusion (Fig. 2a), (b) a patient with a left MCA TIA who already had a minor left MCA stroke with documented occlusion 5 months before (Fig. 3b, (c) a patient with vertebrobasilar TIA with bilateral occlusion documented 4 and 2 years earlier (Fig. $3 c$ and d). The five false-negative results were obtained in five patients with acute MCA stroke, with the right ICA occluded in four and the left in one patient.

We found the ring sign to be present in different pathogenetic mechanisms of occlusion (Fig. 4): local atheromatosis, dissection or cardiac sources, and also in the single patient with occlusion related to sphenoid osteomyelitis.

\section{Discussion}

This study presents CTA-based criteria to classify extracranial internal carotid occlusion in asymptomatic and symp-

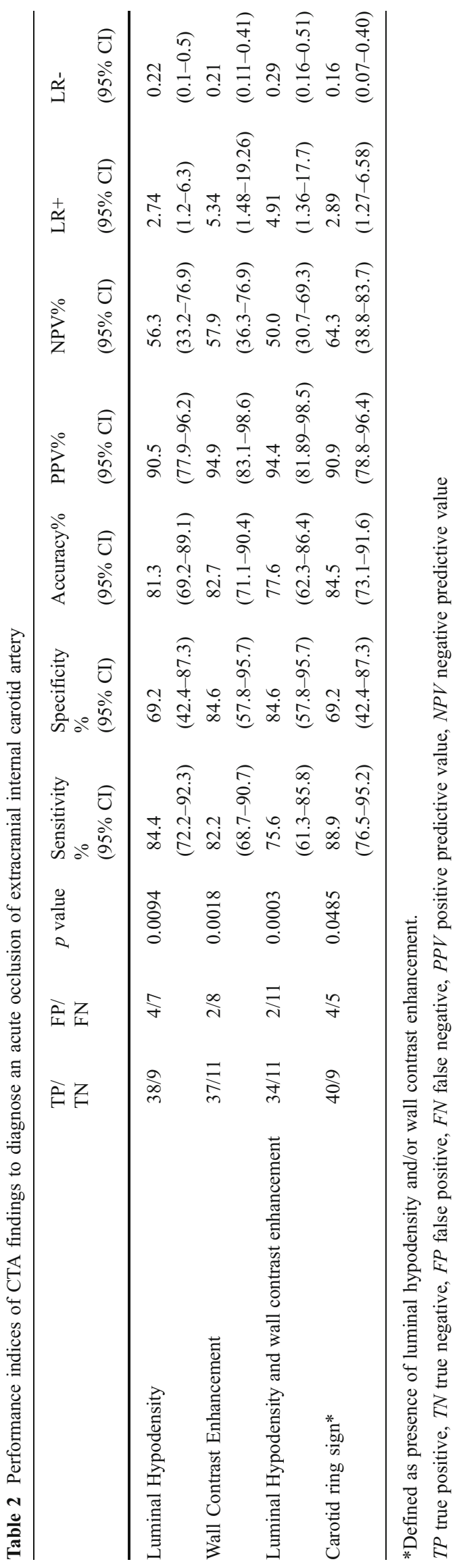



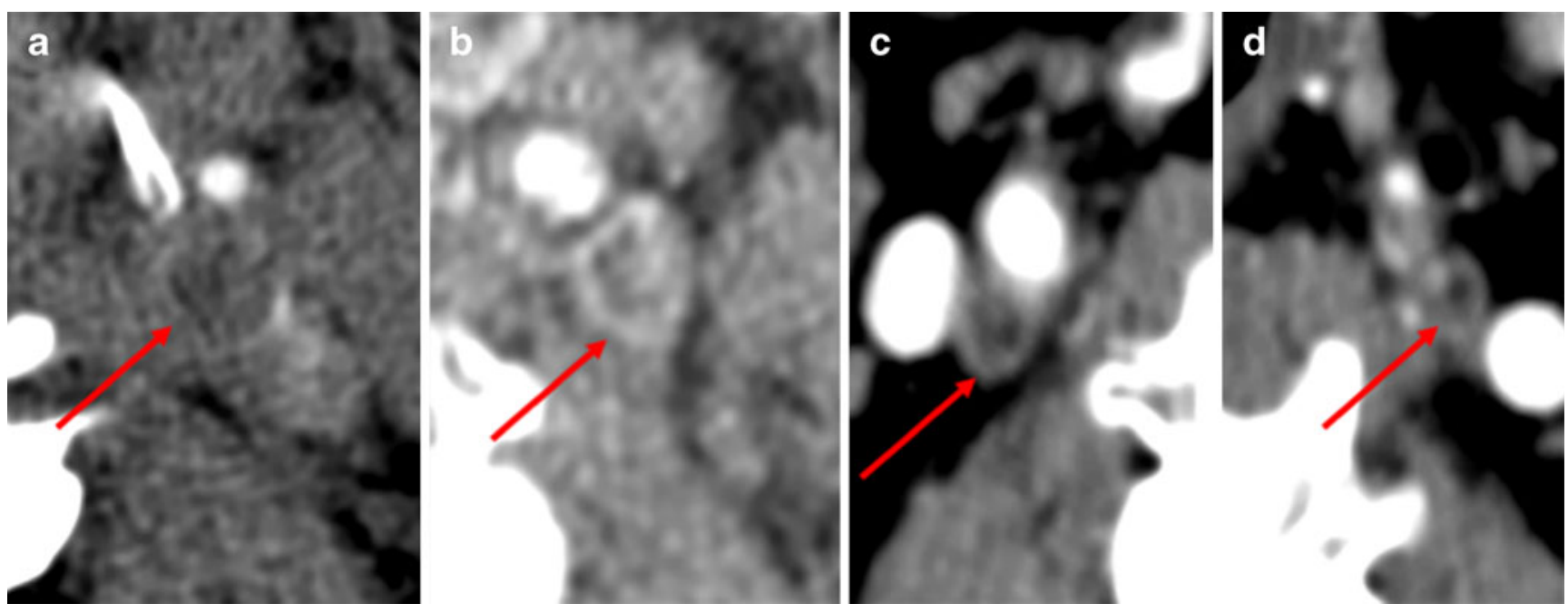

Fig. 3 False-positive results: in all four patients, circumstantial evidence suggested chronic carotid occlusion, but a carotid ring sign was present a a patient with a right MCA stroke during CEA for symptomatic right ICA stenosis with an incidental left ICA occlusion

tomatic patients as acute (within 7 days) or chronic (older than 30 days). On axial CTA sections, the presence of the carotid ring sign - defined as ring-like contrast enhancement in the carotid wall and/or intraluminal hypodensity—reliably differentiated acute from chronic occlusion when compared to circumstantial evidence of the timing of the occlusion.

In patients with recent carotid stent, 3D CTA has been used to follow the sequential change of the intraluminal diameter (a), b a patient with a left MCA TIA who already had a minor left MCA stroke with documented occlusion 5 months before (b), $\mathbf{c}$ a patient with vertebrobasilar TIA with bilateral occlusion documented 4 and 2 years earlier $(\mathbf{c}$ and $\mathbf{d})$

and thrombus formation [12]. On the contrary, in patients presenting with acute stroke and a carotid occlusion, there is no radiological method to reliably estimate the timing of the occlusion. As a result, these are currently classified either as symptomatic or asymptomatic. However, an acute ischemic stroke distal to an occluded ICA is not proof for the latter being the cause of the stroke. Moreover, it may be difficult to decide in a patient with multiple arterial occlusions which
Fig. 4 The carotid ring sign is present in acute internal carotid occlusion independently of the cause of occlusion: patient with a atherosclerosis, b dissection, c osteomyelitis in the sphenoidal bones with retrograde carotid occlusion
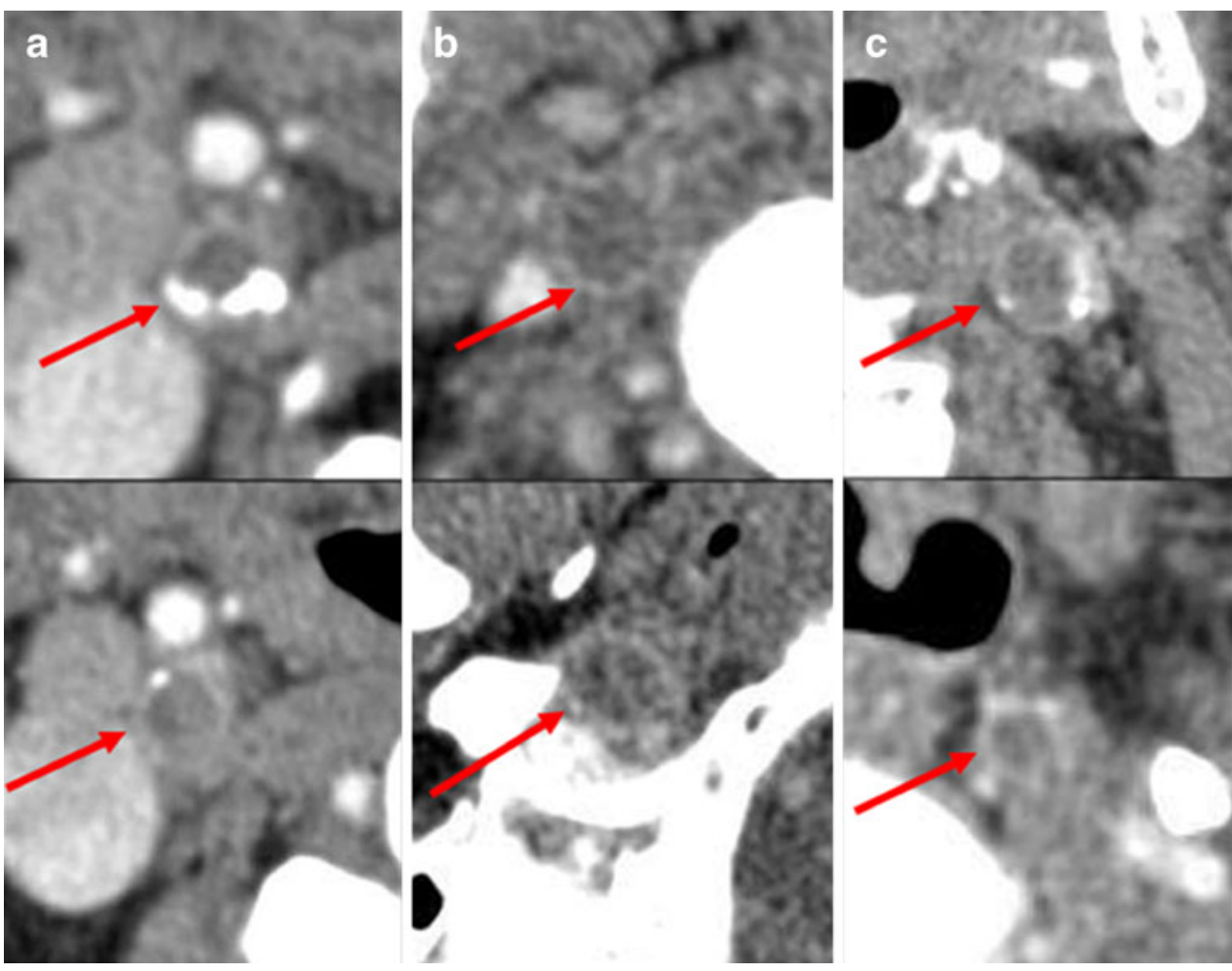

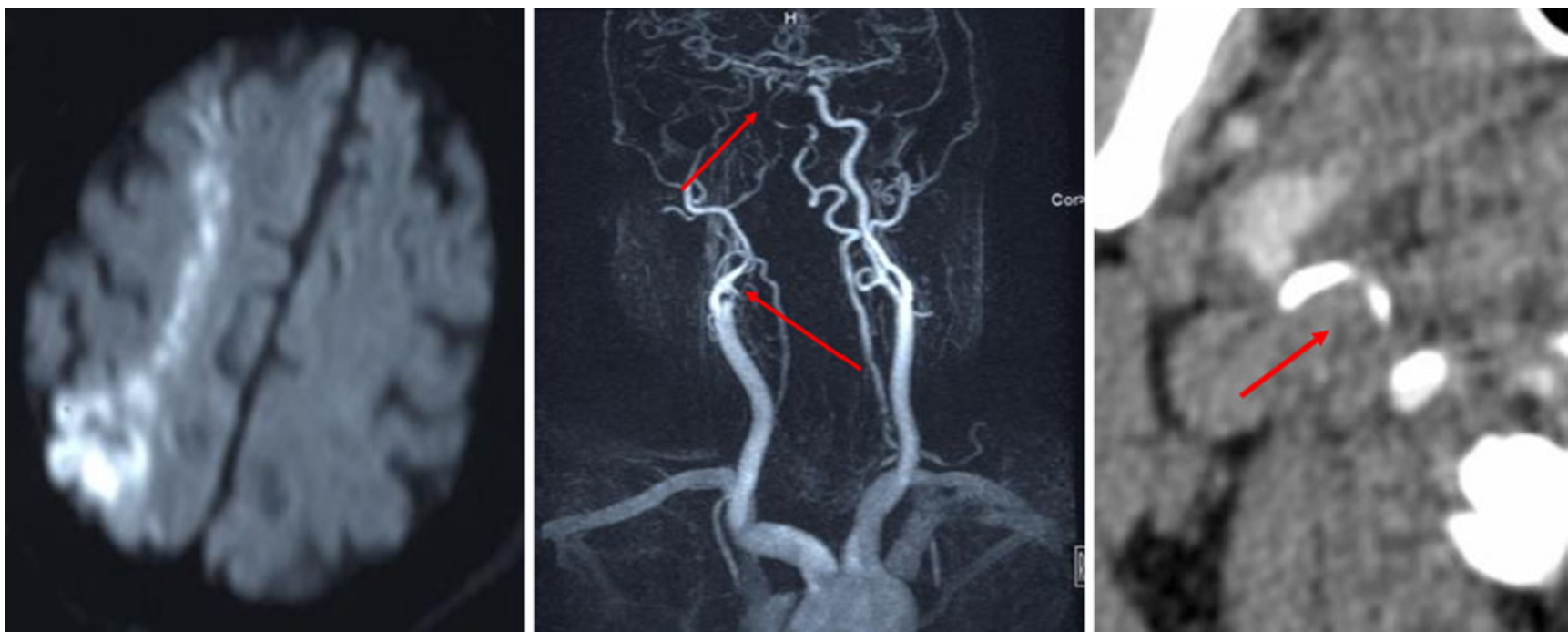

Fig. 5 A 73-year-old man with hypertension and diabetes attended the emergency room due to urinary retention. However, in the emergency room, he developed a rapid decrease of consciousness, left homonymous hemianopsia, conjugate gaze deviation to the right and bilateral, left-predominant hemiplegia. Clinically, both anterior and posterior circulation symptoms were present. Acute CT showed no acute lesions, and CTA revealed occlusion of the right internal carotid (RIC) artery and the basilar artery (BA). Emergent MRI showed acute infarctions in the right hemispheric border zones (left figure), a left thalamic, small left pontine and right superior cerebellar strokes, and confirmed the RIC and BA occlusions (middle figure). Based on the combination of clinical presentation, MRI findings, and the absence of the ring sign on the CT in the RIC (right figure), a decision was made to perform acute intravascular thrombolysis combined with posterior circulation (BA) thromboaspiration and angioplasty. Recovery was slow but steady thereafter, and after a prolonged rehabilitation, patient returned home

vasorum in the viable arterial wall. The luminal hypodensity corresponds to the absence of contrast uptake by the inherently vascular occluding thrombus. In contrast, the arterial wall may not be well perfused anymore in chronic occlusion with scarification of the thrombus and vessel. Similar signs were previously described for the diagnosis of internal jugular venous thrombosis $[13,14]$, as well as deep venous thrombosis of the lower limbs and the pelvic region [15]. However, attention should be drawn for conditions which may mimic the carotid ring sign such as calcified plaques, floating thrombus (also called the "donut sign" the ring sign may be the contrast enhancement of the vasa
A pathophysiological explanation for the occurrence of

one caused acute stroke symptoms, particularly if acute ischemia is demonstrated in border zone regions. Hence, it may be impossible to decide which artery should be acutely treated. The presence or absence of the carotid ring sign, in combination with circumstantial clinical evidence, may allow identifying more efficiently the truly symptomatic lesions. Such a patient case is described in Fig. 5, where the absence of the ring sign was used along with information available from other investigations and clinical facts.
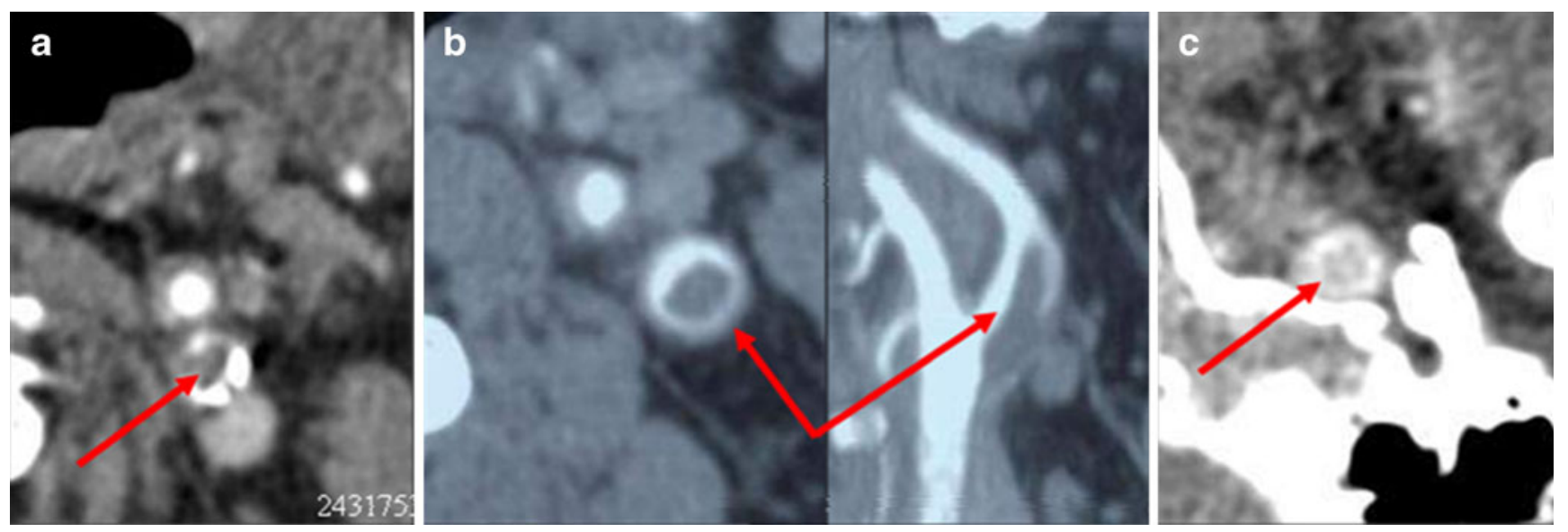

Fig. 6 Conditions which may mimic the carotid ring sign: a calcified plaques in the carotid wall, $\mathbf{b}$ floating thrombus, $\mathbf{c}$ calcified jugular vein 
[16]), calcified jugular vein (Fig. 6). Also, it is possible that the sign does not differentiate well between acute and subacute occlusions, as was shown by our two false-positive results, both of which were actually subacute occlusions (first diagnosed at 2 weeks and 5 months before CTA).

This study is subject to limitations; we had to use circumstantial evidence to classify carotid occlusions as acute or chronic, since there is still no gold standard diagnostic test. It is possible that in some instances, this evidence lead us to wrong estimation of the true timing of the occlusion; therefore, it is possible that some of the predictions of the carotid ring sign, which were considered false, where in fact, the available circumstantial evidence classified the timing of the occlusion incorrectly.

In conclusion, the carotid ring sign on CTA helps to differentiate acute from chronic carotid occlusion. If confirmed by further studies, this information may be helpful in studying ischaemic symptoms and selecting treatment strategies in patients with carotid occlusions.

Conflict of interest We declare that we have no conflict of interest.

\section{References}

1. Adams HP Jr, Bendixen BH, Leira E, Chang KC, Davis PH, Woolson RF, Clarke WR, Hansen MD (1999) Antithrombotic treatment of ischemic stroke among patients with occlusion or severe stenosis of the internal carotid artery: a report of the Trial of Org 10172 in Acute Stroke Treatment (TOAST). Neurology $53: 122-125$

2. De Silva DA, Brekenfeld C, Ebinger M, Christensen S, Barber PA, Butcher KS, Levi CR, Parsons MW, Bladin CF, Donnan GA, Davis SM (2010) The benefits of intravenous thrombolysis relate to the site of baseline arterial occlusion in the Echoplanar Imaging Thrombolytic Evaluation Trial (EPITHET). Stroke 41:295-299

3. Linfante I, Llinas RH, Selim M, Chaves C, Kumar S, Parker RA, Caplan LR, Schlaug G (2002) Clinical and vascular outcome in internal carotid artery versus middle cerebral artery occlusions after intravenous tissue plasminogen activator. Stroke 33:2066-2071
4. Rob CG (1969) Operation for acute completed stroke due to thrombosis of the internal carotid artery. Surgery 65:862-865

5. Mourand I, Brunel H, Vendrell JF, Thouvenot E, Bonafe A (2010) Endovascular stent-assisted thrombolysis in acute occlusive carotid artery dissection. Neuroradiology 52:135-140

6. Jovin TG, Gupta R, Uchino K, Jungreis CA, Wechsler LR, Hammer MD, Tayal A, Horowitz MB (2005) Emergent stenting of extracranial internal carotid artery occlusion in acute stroke has a high revascularization rate. Stroke 36:2426-2430

7. Aleksic M, Rueger MA, Lehnhardt FG, Sobesky J, Matoussevitch V, Neveling M, Heiss WD, Brunkwall J, Jacobs AH (2006) Primary stroke unit treatment followed by very early carotid endarterectomy for carotid artery stenosis after acute stroke. Cerebrovasc Dis 22:276-281

8. Flint AC, Duckwiler GR, Budzik RF, Liebeskind DS, Smith WS (2007) Mechanical thrombectomy of intracranial internal carotid occlusion: pooled results of the MERCI and Multi MERCI Part I trials. Stroke 38:1274-1280

9. Nedeltchev K, Brekenfeld C, Remonda L, Ozdoba C, Do DD, Arnold M, Mattle HP, Schroth G (2005) Internal carotid artery stent implantation in 25 patients with acute stroke: preliminary results. Radiology 237:1029-1037

10. Sugg RM, Malkoff MD, Noser EA, Shaltoni HM, Weir R, Cacayorin ED, Grotta JC (2005) Endovascular recanalization of internal carotid artery occlusion in acute ischemic stroke. AJNR Am J Neuroradiol 26:2591-2594

11. Michel P, Odier C, Rutgers M, Reichhart M, Maeder P, Meuli R, Wintermark M, Maghraoui A, Faouzi M, Croquelois A, Ntaios G (2010) The Acute Stroke Registry and Analysis of Lausanne (ASTRAL): design and baseline analysis of an ischemic stroke registry including acute multimodal imaging. Stroke 41:24912498

12. Watarai H, Kaku Y, Yamada M, Kokuzawa J, Tanaka T, Andoh T, Iwama T (2009) Follow-up study on in-stent thrombosis after carotid stenting using multidetector CT angiography. Neuroradiology 51:243-251

13. Chowdhury K, Bloom J, Black MJ, al-Noury K (1990) Spontaneous and nonspontaneous internal jugular vein thrombosis. Head Neck 12:168-173

14. Cohen JP, Persky MS, Reede DL (1985) Internal jugular vein thrombosis. Laryngoscope 95:1478-1482

15. Langer B, Kauffman P, Bechara M, Aguiar ET, Aun R (1991) Diagnosis of deep venous thrombosis of the lower limbs by computed tomography. Rev Paul Med 109:149-152

16. Menon BK, Singh J, Al-Khataami A, Demchuk AM, Goyal M (2010) The donut sign on CT angiography: an indicator of reversible intraluminal carotid thrombus? Neuroradiology 52:1055-1056 